\title{
Multiuser MIMO Precoding with Per-Antenna Continuous-Time Constant-Envelope Constraints
}

Christopher Mollén and Erik G Larsson

\author{
Linköping University Post Print
}

Tweet

N.B.: When citing this work, cite the original article.

C2015 IEEE. Personal use of this material is permitted. However, permission to reprint/republish this material for advertising or promotional purposes or for creating new collective works for resale or redistribution to servers or lists, or to reuse any copyrighted component of this work in other works must be obtained from the IEEE:

Christopher Mollén and Erik G Larsson, Multiuser MIMO Precoding with Per-Antenna Continuous-Time Constant-Envelope Constraints, 2015, IEEE International Workshop on Signal Processing Advances in Wireless Communications.

http://dx.doi.org/10.1109/SPAWC.2015.7227040

Postprint available at: Linköping University Electronic Press

http://urn.kb.se/resolve?urn=urn:nbn:se:liu:diva-120251 


\title{
Multiuser MIMO Precoding with Per-Antenna Continuous-Time Constant-Envelope Constraints
}

\author{
Christopher Mollén and Erik G. Larsson \\ Dept. of Electrical Engineering (ISY), Linköping University, 58183 Linköping, Sweden \\ Email: christopher.mollen@liu.se, erik.g.larsson@liu.se
}

\begin{abstract}
A transmission scheme for the multiuser MIMo downlink, where the transmit signal from each antenna has constant envelope and a limited bandwidth, is proposed in order to enable the use of highly efficient, nonlinear amplifiers at the base station. To evaluate its performance, an achievable rate is derived and the necessary transmit power of the proposed scheme is computed for a system with 40 antennas that serves 4 users at data rates around 1 bpcu. For this system and $40 \%$ excess $30 \mathrm{~dB}$-bandwidth, approximately $3 \mathrm{~dB}$ more transmit power is required to achieve the same sum-rate as without the constantenvelope constraints.
\end{abstract}

Index Terms-massive MIMO, multiuser precoding, constantenvelope transmission, PAR.

\section{INTRODUCTION}

The benefits of base stations with large numbers of antennas, so called massive MIMO (Multiple-Input-Multiple-Output) base stations, have proven to be many. Their ability a) to pre-equalize a frequency selective channel will simplify user equipment, b) to do multiuser precoding to achieve a great multiplexing gain will increase spectral efficiency, and c) to make signals coherently add up and deliver a big array powergain will increase the radiated energy efficiency of the overall system. These are just a few of the reasons that massive MIMO is considered to become a key component in the next generation of wireless communication [1].

Yet another benefit of the great number of antennas is the unique opportunity to perform signal shaping in the downlink [2], [3]. Signal shaping is facilitated by the large nullspace of the channel: with $M$ antennas serving $K$ users, any component placed in the $(M-K)$-dimensional nullspace of the channel is invisible to the users. For example, the transmit signal can be augmented by a component that lies in the channel nullspace such that the discrete-time effective transmitted signal has low peak-to-average ratio [3], or even constant envelope [2]. However, after pulse shape filtering, both these methods still yield waveforms with peak-to-average ratios greater than $4 \mathrm{~dB}$.

This paper studies the massive MIMO channel with constantenvelope constraints on the emitted continuous-time waveforms, i.e. with stricter constraints than in [2], [3], where only the discrete-time signals before pulse shape filtering were constrained. A lower bound on the downlink sum-capacity is given by devising a transmission scheme and computing its sum-rate. In the proposed scheme, each base station antenna emits a constant-envelope signal $(0 \mathrm{~dB}$ peak-to-average ratio) and the users concurrently receive arbitrary variable-envelope symbols. With such transmit signals, no linearity requirements on the hardware of the base station are needed and the power amplifiers can be operated at maximum efficiency.

Note that continuous-phase modulation for SISO systems is a well-studied research field, see e.g. [4], [5]. The possibility to use continuous-time constant-envelope signals in point-topoint MIMO systems has also been investigated before. In [6], [7] for example, achievable data rates for different constantenvelope symbol constellations are computed. The multiuser MIMO setup considered here, however, is fundamentally different and requires a new approach.

\section{System Model}

The downlink transmission from a base station with $M$ antennas to $K$ single-antenna users will be studied. Let $x_{m}(t)$ be the complex baseband transmit signal from antenna $m$. Then the received signal $r_{k}(t)$ at user $k$ is given by

$$
r_{k}(t)=\sqrt{P} \sum_{m=1}^{M}\left(h_{k m}(\tau) \star x_{m}(\tau)\right)(t)+w_{k}(t),
$$

where $h_{k m}(\tau)$ is the impulse response of the channel between antenna $m$ and user $k, w_{k}(t)$ is a complex white Gaussian noise process with zero mean and spectral height $N_{0}$ that is independent of the transmit signals $\left\{x_{m}(t)\right\}$ and the channel $\left\{h_{k m}(\tau)\right\}$. The power of the transmit signals should fulfill

$$
\mathrm{E}\left[\left|x_{m}(t)\right|^{2}\right]=\frac{1}{M} \text {. }
$$

The factor $P$ therefore represents the total radiated power.

Each user is equipped with a filter with impulse response $p(\tau)$ that is chosen such that

$$
\int_{-\infty}^{\infty}|p(\tau)|^{2} \mathrm{~d} \tau=1 / T
$$

The received signal is filtered by $p(\tau)$ and uniformly sampled to produce the received samples

$$
r_{k}[n] \triangleq y_{k}[n]+w_{k}[n], \quad n=0, \ldots, N-1 .
$$

Each of these samples is the sum of two parts: the noise-free signal and a noise sample that is independent of the signal

$$
\begin{aligned}
& y_{k}[n] \triangleq \sqrt{P} \int_{-\infty}^{\infty} p(\tau) \sum_{m=1}^{M}\left(h_{k m}(t) \star x_{m}(t)\right)(n T-\tau) \mathrm{d} \tau, \\
& w_{k}[n] \triangleq \int_{-\infty}^{\infty} p(\tau) w_{k}(n T-\tau) \mathrm{d} \tau .
\end{aligned}
$$




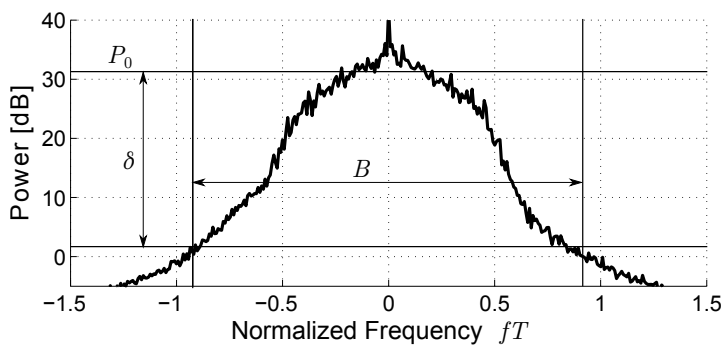

Fig. 1. The power spectral density of a typical constant-envelope signal. Its $30 \mathrm{~dB}$-bandwidth $B T=1.8$ is indicated.

Each user thus observes $N$ samples. The sampling period $T$ will be referred to as the symbol period. It is assumed that the impulse response $p(\tau)$ is a root-Nyqvist pulse of period $T$; then the noise samples are i.i.d. $\mathcal{C N}\left(0, N_{0} / T\right)$.

\section{The Constant-EnVELope MIMO CHANNEL}

Let the transmit signals $\left\{x_{m}(t)\right\}$ be stochastic processes and assume that they are of some operational power spectral density $S_{x}(f)$ [8].

Definition 1: A continuous-time constant-envelope signal is a stochastic process that fulfills

$$
\left|x_{m}(t)\right|^{2}=\frac{1}{M}, \quad \forall t,
$$

almost surely.

Since the only strictly bandlimited signals that have property (7) are pure sinusoids, a relaxed measure of bandwidth will be used.

Definition 2: The $\delta$-bandwidth with respect to the symbol rate $1 / T$ of the process $x_{m}(t)$ is

$$
B \triangleq \inf \left\{B^{\prime} \geq 0: S_{x}(f)<P_{0} / \delta, \quad \forall|f|>B^{\prime} / 2\right\},
$$

where the in-band power is given by

$$
P_{0} \triangleq T \int_{-1 /(2 T)}^{0^{-}} S_{x}(f) \mathrm{d} f+T \int_{0^{+}}^{1 /(2 T)} S_{x}(f) \mathrm{d} f .
$$

The power at $f=0$ is excluded in the in-band power to allow for signals with a nonzero mean. The $30 \mathrm{~dB}$-bandwidth measure is illustrated in Figure 1. The fraction of the bandwidth greater than the symbol rate

$$
T B-1,
$$

will be referred to as the excess $\delta$-bandwidth.

Now the channel that is studied in this paper can be defined in terms of the two previous definitions.

Definition 3: The $M \times K$ continuous-time constant-envelope MIMO broadcast channel of $\delta$-bandwidth $B_{\max }$ is the channel described in (1) between $M$ antennas, which only emit continuous-time constant-envelope transmit signals $\left\{x_{m}(t)\right\}$ with $\delta$-bandwidth smaller than or equal to $B_{\max }$, and each of the $K$ single-antenna users that receive the signals $\left\{r_{k}(t)\right\}$.

\section{The Continuous-Time Constant-Envelope MASSIVE MIMO DOWNLINK}

To lower-bound the sum-capacity of the constant-envelope channel detailed in Section III, a transmission scheme for a massive MIMO downlink that uses transmit signals with constant envelopes is presented here. The proposed precoder of this downlink will be called the Continuous-Time ConstantEnvelope (CTCE) precoder.

\section{A. CTCE Precoding}

The random symbol intended for user $k$ at sample instant $n$ is denoted by $u_{k}[n]$, for all $k=1, \ldots, K$ and $n=$ $0, \ldots, N-1$. The symbols are required to have unit energy

$$
\mathrm{E}\left[\left|u_{k}[n]\right|^{2}\right]=1, \quad \forall n, k .
$$

Given the parameters $\gamma, \lambda_{1}, \lambda_{2} \in \mathbb{R}^{+}$, we choose the transmit signals, for each realization of the channel and the random symbols, to be a continuous solution to

$$
\begin{aligned}
& \min _{\left\{x_{m}(t)\right\}}\left(\sum_{k=1}^{K} \sum_{n=0}^{N-1}\left|y_{k}[n]-\sqrt{\gamma P} u_{k}[n]\right|^{2}\right. \\
& \left.+\lambda_{1} \sum_{m=1}^{M} \int_{-\infty}^{\infty}\left|\frac{\mathrm{d}}{\mathrm{d} t} x_{m}(t)\right|^{2} \mathrm{~d} t+\lambda_{2} \sum_{m=1}^{M} \int_{-\infty}^{\infty}\left|\frac{\mathrm{d}^{2}}{\mathrm{~d} t^{2}} x_{m}(t)\right|^{2} \mathrm{~d} t\right)
\end{aligned}
$$

subject to the modulus constraint $\left|x_{m}(t)\right|=1 / \sqrt{M}, \forall m, t$.

The precoder given by (12) minimizes the mismatch between the actual received sample and the desired symbol and lets each user receive a new symbol every instant $t=n T$. The power of the desired symbols is determined by the parameter $\gamma$. The two latter terms in (12) serve the purpose of regularizing the first and second derivatives of the transmit signals in order to produce smooth signals. By choosing the regularizing factors $\lambda_{1}$ and $\lambda_{2}$ large enough, it has been observed that the resulting solution has a limited $\delta$-bandwidth. The parameters $\gamma, \lambda_{1}$ and $\lambda_{2}$ will be chosen to maximize the sum-rate and to fulfill a bandwidth requirement; see Section V.

The optimization problem (12) can be approximately solved in discrete time by expressing each $y_{k}[n]$ in terms of sampled versions of the transmit signals $\left\{x_{m}(t)\right\}$. If the sampling rate is high enough, there are constant-envelope modulation schemes that produce continuous-time signals with limited bandwidth from the discrete-time solution, see Section IV-B.

The noise-free received sample in (5) can be rewritten as:

$$
y_{k}[n]=\sqrt{P} \sum_{m=1}^{M} \int \underbrace{\int p(\check{\tau}) h_{k m}(\tau-\check{\tau}) \mathrm{d} \check{\tau}}_{\triangleq f_{k m}(\tau)} x_{m}(n T-\tau) \mathrm{d} \tau .
$$

The inner integral $f_{k m}(\tau) \triangleq\left(p(t) \star h_{k m}(t)\right)(\tau)$ could be estimated by letting the users send uplink pilots. Here, however, it is assumed that $f_{k m}(\tau)$ is perfectly known by the base station.

Denote the $\kappa$-times oversampled (with respect to the symbol period $T$ ) signals

$$
\begin{gathered}
x_{m}[\nu] \triangleq x_{m}(\nu T / \kappa), \\
f_{k m}[\nu] \triangleq \frac{T}{\kappa} f_{k m}(\nu T / \kappa) .
\end{gathered}
$$


It is assumed that there exist integers $\ell_{\min }$ and $\ell_{\max }$ such that $f_{k m}(\tau)$ practically is zero for $\tau$ outside $\left[\ell_{\min } T / \kappa, \ell_{\max } T / \kappa\right]$. In what follows, only the samples $f_{k m}[\nu]$ with indices $\nu \in$ $\left[\ell_{\min }, \ell_{\max }\right]$ will be considered.

The aggregate channel impulse response $f_{k m}(\tau)$ is bandlimited to $\kappa / T$ if the impulse response $p(\tau)$ is. Assume that the transmit signal $x_{m}(t)$ is bandlimited to $\kappa / T$ too; then (13) can be written in terms of the two discrete-time signals $x_{m}[\nu]$ and $f_{k m}[\nu]$. Even if the modulated transmit signal, being a constant-envelope signal with non-constant phase derivative, is not strictly bandlimited, it is practically bandlimited to $\kappa / T$ for some $\kappa$ when the parameters $\lambda_{1}$ and $\lambda_{2}$ are large enough, as will be shown in Section VI. Therefore by choosing $\kappa$ big enough, the received signal is approximately given by

$$
y_{k}[n] \approx \sqrt{P} \sum_{m=1}^{M} \sum_{\ell=\ell_{\min }}^{\ell_{\max }} f_{k m}[\ell] x_{m}[n \kappa-\ell] .
$$

Denote by $\zeta_{k}[n]$ the argument of the modulus operator in the first term in (12) (divided by $\sqrt{P}$ ):

$$
\zeta_{k}[n]=\sqrt{\gamma} u_{k}[n]-\sum_{m=1}^{M} \sum_{\ell=\ell_{\min }}^{\ell_{\max }} f_{k m}[\ell] x_{m}[n \kappa-\ell] .
$$

By using the first-order approximations of the first and second derivatives

$$
\begin{aligned}
&\left.\frac{\mathrm{d}}{\mathrm{d} t} x_{m}(t)\right|_{t=\frac{\nu T}{\kappa}} \approx \frac{x_{m}[\nu-1]-x_{m}[\nu]}{T / \kappa} \triangleq x_{m}^{\prime}[\nu] \\
&\left.\frac{\mathrm{d}^{2}}{\mathrm{~d} t^{2}} x_{m}(t)\right|_{t=\frac{\nu T}{\kappa}} \approx \frac{x_{m}[\nu-1]-2 x_{m}[\nu]+x_{m}[\nu+1]}{(T / \kappa)^{2}} \triangleq x_{m}^{\prime \prime}[\nu],
\end{aligned}
$$

the optimization problem (12) can be approximated as

$$
\min _{\left|x_{m}[\nu]\right|=\frac{1}{\sqrt{M}}} \sum_{k, n}\left|\zeta_{k}[n]\right|^{2}+\lambda_{1} \sum_{m, \nu}\left|x_{m}^{\prime}[\nu]\right|^{2}+\lambda_{2} \sum_{m, \nu}\left|x_{m}^{\prime \prime}[\nu]\right|^{2} .
$$

Only the transmit samples $x_{m}[\nu]$ with indices

$$
\nu=-\ell_{\max }, \ldots,(N-1) \kappa-\ell_{\min }
$$

influence the received samples. These are the samples that are optimized with respect to in (19).

The objective function in (19) is non-convex and the optimization is hard to solve explicitly. By using a technique similar to the one used in [2], a solver that uses cyclic optimization can be devised by observing that the problem can be explicitly solved for one sample $x_{\tilde{m}}[\tilde{\nu}]$ by

$$
x_{\tilde{m}}[\tilde{\nu}]=\frac{1}{\sqrt{M}} \frac{z_{\tilde{m}}^{*}[\tilde{\nu}]}{\left|z_{\tilde{m}}[\tilde{\nu}]\right|},
$$

where $z_{\tilde{m}}[\tilde{\nu}] \triangleq z_{1}+z_{2}+z_{3}$ is the sum of the three terms

$$
\begin{aligned}
& z_{1} \triangleq \sum_{k=1}^{K} \sum_{n=\underline{n}}^{\bar{n}} f_{k \tilde{m}}^{*}[n \kappa-\tilde{\nu}]\left(\zeta_{k}[n]+f_{k \tilde{m}}[n \kappa-\tilde{\nu}] x_{\tilde{m}}[\tilde{\nu}]\right) \\
& z_{2} \triangleq \frac{\lambda_{1} \kappa^{2}}{T^{2}}\left(x_{\tilde{m}}^{*}[\tilde{\nu}-1]+x_{\tilde{m}}^{*}[\tilde{\nu}+1]\right), \\
& z_{3} \triangleq \frac{\lambda_{2} \kappa^{4}}{T^{4}}\left(4 x_{m}^{*}[\nu-1]-x_{m}^{*}[\nu-2]+4 x_{m}^{*}[\nu+1]-x_{m}^{*}[\nu+2]\right) .
\end{aligned}
$$

The limits in (22) are given by

$$
\begin{aligned}
& \underline{n}=\max \left(\left\lceil\left(\ell_{\min }+\tilde{\nu}\right) / \kappa\right\rceil, 0\right), \\
& \bar{n}=\min \left(\left\lfloor\left(\ell_{\max }+\tilde{\nu}\right) / \kappa\right\rfloor, N-1\right) .
\end{aligned}
$$

Since the objective function in (19) does not increase when a signal sample $x_{\tilde{m}}[\tilde{\nu}]$ is set to its optimum value (21), an algorithm that sets the signal samples one-by-one to their optimal values by letting the indices cyclically run through

$$
\begin{gathered}
(\tilde{m}, \tilde{\nu}):\left(1,-\ell_{\max }\right) \rightarrow\left(2,-\ell_{\max }\right) \rightarrow \cdots \rightarrow\left(M,-\ell_{\max }\right) \\
\rightarrow\left(1,1-\ell_{\max }\right) \rightarrow \cdots \rightarrow\left(M, 1-\ell_{\max }\right) \\
\rightarrow \cdots \cdots \rightarrow\left(M,(N-1) \kappa-\ell_{\min }\right)
\end{gathered}
$$

a couple of rounds will make the objective function (19) converge to a local minimum. How many cycles are needed depends on the parameters $\lambda_{1}$ and $\lambda_{2}$. For small $\lambda_{1}$ and $\lambda_{2}$, which correspond to lax bandwidth requirements, the optimization converges in 5-10 rounds. The greater $\lambda_{1}$ and $\lambda_{2}$, and narrower bandwidths, the more rounds are needed. To produce steep and narrow spectra for tough bandwidth requirements, as many as 100 rounds might be needed. How close the local minimum, which the algorithm converges to, is to the global depends on the initialization of the samples.

Many initialization methods have been tested, such as: setting $x_{m}[\nu]=0$ at first, to initialize $x_{m}[\nu]$ to have the same phase for all $m$ and $\nu$, to initialize with random phases, etc. The best method that was found, which is the method later used in Section VI, is to successively increase the oversampling factor $\kappa$. An initial optimization is done with oversampling factor $\kappa_{0} \triangleq 1$ over the signals $\left\{x_{m}^{(0)}[\nu] \triangleq x_{m}\left(\nu T / \kappa_{0}\right)\right\}$ by initializing $x_{m}^{(0)}[\nu]=1 / \sqrt{M}$. The so-obtained solution is used to initialize a second optimization over the $\kappa_{1} \triangleq 2$-times oversampled signals $x_{m}^{(1)}[\nu] \triangleq x_{m}\left(\nu T / \kappa_{1}\right)$. The result is again used to initialize a further optimization with a higher oversampling factor. The $i$-th optimization is done over the $\kappa_{i} \triangleq 2^{i}$-times oversampled signals

$$
x_{m}^{(i)}[\nu] \triangleq x_{m}\left(\nu T / \kappa_{i}\right)
$$

that are initialized by

$x_{m}^{(i)}[\nu]= \begin{cases}x_{m}^{(i-1)}[\nu / 2], & \text { if } \nu \text { is even, } \\ \frac{1}{\sqrt{M}} \frac{x_{m}^{(i-1)}[(\nu-1) / 2]+x_{m}^{(i-1)}[(\nu+1) / 2]}{\left|x_{m}^{(i-1)}[(\nu-1) / 2]+x_{m}^{(i-1)}[(\nu+1) / 2]\right|}, & \text { if } \nu \text { is odd. }\end{cases}$

The optimization procedure is terminated after a high enough oversampling factor is reached, e.g. $\kappa_{3}=8$. A high enough oversampling factor a) ensures that the signals maintain their limited bandwidths after constant-envelope modulation and b) does not improve the cost function much from the previous oversampling factor.

\section{B. Constant-Envelope Modulation}

A discrete-time constant-envelope signal can be modulated into a continuous-time constant-envelope signal by $x_{m}(t)=$

$$
\frac{1}{\sqrt{M}} \exp \left(j \int_{-\infty}^{t} \sum_{\nu=-\ell_{\max }}^{(N-1) \kappa-\ell_{\min }} \arg \left(x_{m}^{*}[\nu-1] x_{m}[\nu]\right) p_{f}\left(\tau-\nu \frac{T}{\kappa}\right) \mathrm{d} \tau\right),
$$


where $-\pi<\arg (z) \leq \pi$ is the principal argument of $z$, and $p_{f}(\tau)$ is an $L^{2}$-function called the frequency shaping pulse that satisfies

$$
\int_{-\infty}^{\infty} p_{f}(\tau) \mathrm{d} \tau=1
$$

For example, to get linear interpolation of the phase, the frequency shaping pulse shall be chosen as

$$
p_{f}(\tau)=\left\{\begin{array}{ll}
\frac{\kappa}{T}, & -\frac{T}{\kappa} \leq \tau \leq 0 \\
0, & \text { otherwise }
\end{array} .\right.
$$

For other choices of frequency shaping pulses, see e.g. [5]. Let $B_{\text {disc }}$ be the $\delta$-bandwidth of the signal obtained from ideal pulse-amplitude modulation (with $\operatorname{sinc}(t \kappa / T)$ ) of the discrete-time signal $x_{m}[\nu]$. The modulation scheme in (28) ensures a continuous-phase constant-envelope signal, whose $\delta$-bandwidth is approximately $B_{\text {disc }}$, if the oversampling factor $\kappa \gg B_{\text {disc }} T$ is big relative to the bandwidth.

Because the discrete-time signals of the CTCE precoder are highly oversampled however, no choice of the frequency shaping pulse results in significantly lower bandwidth than what is obtained from linear interpolation of the phase. Therefore, linear interpolation is good enough to use.

\section{Achievable Rate}

The $n$-th received sample at user $k$, same as (4), can always be written as the sum of three terms:

$$
r_{k}[n]=\sqrt{P} g_{k} u_{k}[n]+\sqrt{P} i_{k}[n]+w_{k}[n] .
$$

The first term is the desired signal, scaled by some deterministic factor $g_{k}$. The second is an error term, that describes the mismatch between the desired signal and the noise-free received signal. The third is a noise term.

The deterministic factor is chosen to be

$$
g_{k}=\frac{1}{\sqrt{P}} \mathrm{E}\left[u_{k}^{*}[n] r_{k}[n]\right],
$$

in order to make the interference and symbol terms uncorrelated, $\mathrm{E}\left[u_{k}^{*}[n] i_{k}[n]\right]=0$. Because the interference is uncorrelated to the symbol, assuming that it is Gaussian distributed is to assume a worst-case scenario. The rate

$$
\begin{gathered}
R_{k} \triangleq \log _{2}\left(1+\frac{P G_{k}}{P I_{k}+N_{0} / T}\right)\left[\begin{array}{c}
\text { bpcu } \\
\text { (bits-per-channel-use) }
\end{array}\right] \\
G_{k} \triangleq\left|g_{k}\right|^{2}, \quad I_{k} \triangleq \mathrm{E}\left[\left|i_{k}[n]\right|^{2}\right] .
\end{gathered}
$$

is thus achievable with Gaussian distributed symbols. The same bound is derived in [9] for point-to-point MIMO systems. The expectations in (31) and in (33) are taken with respect to all sources of randomness: channel, symbols and noise.

Both the gain $G_{k}$ and the interference $I_{k}$ depend on the parameters $\gamma, \lambda_{1}$ and $\lambda_{2}$ and on the distribution of the symbols. By maximizing (32) with respect to $\gamma, \lambda_{1}, \lambda_{2}$, an achievable sum-rate for CTCE precoding can be established:

$$
R_{\mathrm{CTCE}} \triangleq \max _{\left\{\left(\gamma, \lambda_{1}, \lambda_{2}\right): B \leq B_{\max }\right\}} \sum_{k=1}^{K} R_{k}\left(\gamma, \lambda_{1}, \lambda_{2}\right),
$$

TABLE I

SIMULATION SETUP

\begin{tabular}{ll}
\hline Baudrate & $1 / T=5 \times 10^{6} \mathrm{~Hz}$ \\
Maximum excess delay & $\sigma_{\tau}=3 \times 10^{-6} \mathrm{~s} \quad(=15 T)$ \\
No. propagation paths & $D=10$ \\
Receive filter $p(t)$ & root-raised cosine, roll-off 0.22 \\
Symbol constellation & i.i.d. Gaussian, $u_{k}[n] \sim \mathcal{C N}(0,1)$ \\
Oversampling factor & $\kappa=8$ \\
No. antennas $\times$ users & $M \times K=40 \times 4$ \\
Bandwidth threshold & $\delta=30 \mathrm{~dB}$ \\
\hline
\end{tabular}

where the optimization is over all choices of the parameters $\left(\gamma, \lambda_{1}, \lambda_{2}\right)$ that result in a $\delta$-bandwidth less than a given $B_{\max }$.

\section{Numerical Analysis of the CTCE Precoder}

The performance of the CTCE precoder has been computed through extensive Monte-Carlo simulations. The channel is assumed to be block-fading and modeled in complex baseband by a tapped delay-line. The channel from antenna $m$ to user $k$ is described by the time-invariant impulse response

$$
h_{k m}(\tau)=\sum_{d=1}^{D} \sqrt{a\left(\tau_{d}\right)} \alpha_{d} \delta\left(\tau-\tau_{d}\right),
$$

where $D$ is the number of propagation paths of the channel, $a\left(\tau_{d}\right)$ a power delay profile, $\tau_{d}$ the delay of path $d$ and $\alpha_{d} \in$ $\mathbb{C}$ the phase rotation and small-scale fading of path $d$. The complex attenuations $\left\{\alpha_{d}\right\}$ are modeled as i.i.d. $\mathcal{C N}(0,1)$, and the delays $\left\{\tau_{d}\right\}$ as uniformly distributed between 0 and $\sigma_{\tau}$, where $\sigma_{\tau}$ is the maximum excess delay. The power delay profile is chosen to be

$$
a(\tau)=A e^{-\lambda \tau},
$$

where the decay rate is chosen such that $a\left(\sigma_{\tau}\right)=0.1 A$, and $A$ is chosen such that $\sum_{d=1}^{D} \mathrm{E}\left[a\left(\tau_{d}\right)\right]=1$, i.e.:

$$
\begin{aligned}
& \lambda=\ln (10) / \sigma_{\tau}, \\
& A=\frac{\lambda \sigma_{\tau}}{D\left(1-e^{-\lambda \sigma_{\tau}}\right)} .
\end{aligned}
$$

The studied system is specified in Table I. In Figures 2a and $2 \mathrm{~b}$, the estimated $30 \mathrm{~dB}$-bandwidths of the transmit signals of the CTCE precoder are shown for different choices of the parameters $\gamma, \lambda_{1}$ and $\lambda_{2}$. It can be seen that, for any given $\gamma$, the bandwidth of the transmit signals decreases as the factors $\lambda_{1}$ or $\lambda_{2}$ increase. It can also be seen that when $\gamma$ increases and $\lambda_{1}, \lambda_{2}$ are fixed, the precoder has to put more effort to make the mismatch term in (12) small, which means that the two regularizing terms, together with the bandwidth, will increase.

Compare the bandwidths of the CTCE precoder with the bandwidth $B_{\text {PAM }}$ of a conventional system using pulseamplitude modulation, which ideally would be that of the pulse shaping filter that is matched to the receive filter $p(t)$ (that is $T B_{\mathrm{PAM}}=1.22$ for our choice of receive filter). However, maximum-ratio and zero-forcing precoding produce transmit signals with high peak-to-average ratio that will be subject to 
(a) $\lambda_{1}$ variable and $\lambda_{2}=0$

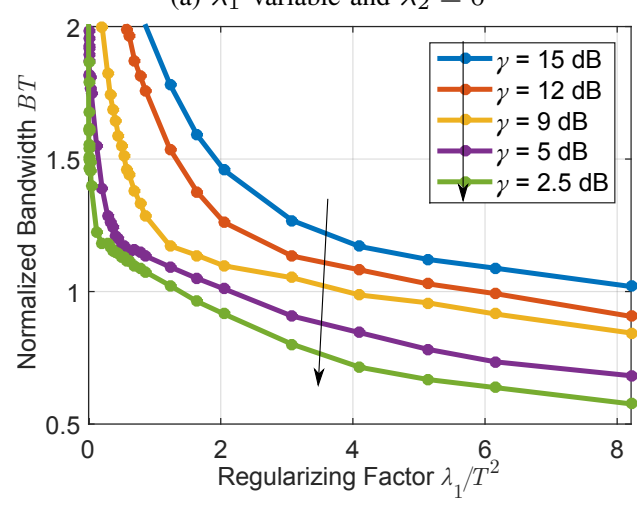

(b) $\lambda_{2}$ variable and $\lambda_{1}=0$

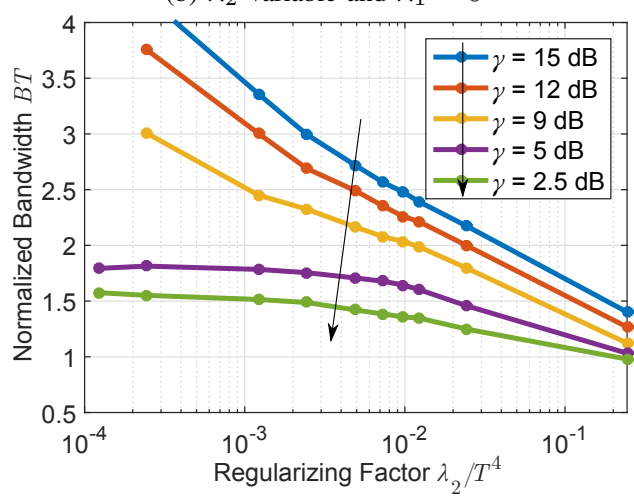

Fig. 2. The $30 \mathrm{~dB}$-bandwidth of CTCE precoded transmit signals for different choices of the regularizing factors $\lambda_{1}$ and $\lambda_{2}$.

spectral regrowth in the power amplifier. Their actual bandwidth is therefore expected to be greater than that of the pulse shaping filter, $T B_{\text {PAM }}>1.22$. How much greater depends on the amount of back-off and the complexity of the amplifiers. Note that CTCE can produce signals with bandwidths narrower than $B_{\text {PAM }}$. However, as seen in Figure $2 \mathrm{a}$, to make the bandwidth narrow, a big regularizing factor $\lambda_{1}$ has to be used, which rapidly reduces the performance.

The sum-rate of the studied system is shown in Figure 3 for different bandwidth requirements $B_{\max }$. The rate was computed by computing $G_{k}$ and $I_{k}$ for a mesh of $\gamma, \lambda_{1}, \lambda_{2}$ and maximizing (34) over the set of parameter values that resulted in a bandwidth smaller than $B_{\max }$. The proposed precoder is compared to conventional maximum-ratio and zero-forcing precoding, for which achievable rates were derived by [10]. If the base station were to use the CTCE precoder instead of these conventional precoders to deliver the same sum-rate, then the its radiated power has to be increased. For $T B_{\max }=1.4$ and for low sum-rates, 2-4 bpcu, this increase is about $3 \mathrm{~dB}$.

It was also noticed in the simulations that the third term in (12), which served the purpose of regularizing the second derivative of the transmit signals, did little to improve the performance. The sum-rate curves in Figure 3 would still be the same if the regularizing term $\lambda_{2}$ were set to zero in (34), with one exception: the sum-rate curve for $T B_{\max }=2$ improves slightly when the second derivative is regularized. It

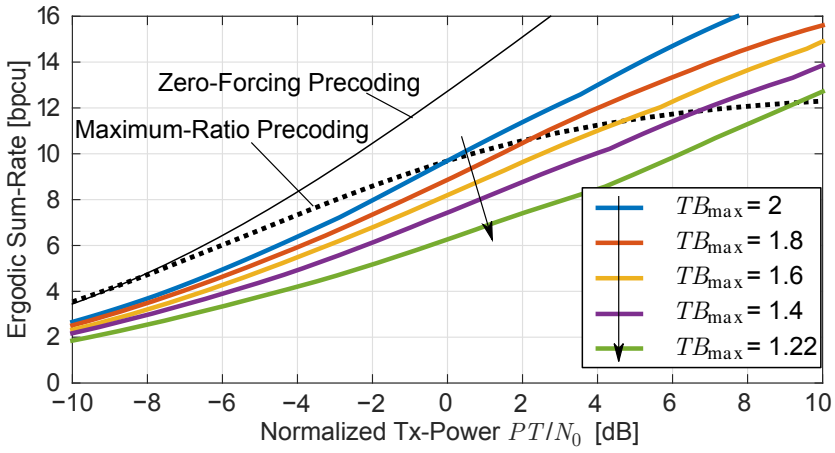

Fig. 3. The ergodic sum-rate of the simulated system for different precoders. The thick lines represent the proposed CTCE precoder for different bandwidths.

thus seems, that adding a smoothing term that regularizes the second derivative to the optimization in (12) is only important if we allow for signals with large excess bandwidths.

\section{CONCLUSION}

We have proposed a massive MIMO downlink, where symbols from arbitrary constellations concurrently are transmitted to multiple users by continuous-time constant-envelopes transmit signals. For low sum-rates, the proposed continuous-time constant-envelope transmission scheme requires approximately $3 \mathrm{~dB}$ more radiated power than conventional linear precoders. However, the proposed system allows for power efficient hardware designs, and its overall net consumed power might therefore be made lower than that of conventional precoders.

\section{ACKNOWLEDGMENT}

The research leading to these results has received funding from the European Union Seventh Framework Programme under grant agreement number ICT-619086 (MAMMOET).

\section{REFERENCES}

[1] E. G. Larsson, O. Edfors, F. Tufvesson, and T. L. Marzetta, "Massive MIMO for next generation wireless systems," IEEE Commun. Mag., vol. 52, no. 2, pp. 186-195, Feb. 2014.

[2] S. Mohammed and E. G. Larsson, "Constant-envelope multi-user precoding for frequency-selective massive MIMO systems," IEEE Wireless Commun. Lett., vol. 2, no. 5, pp. 547-550, Oct. 2013.

[3] C. Studer and E. G. Larsson, "PAR-aware large-scale multi-user MIMOOFDM downlink," IEEE Select. Areas in Commun., vol. 31, no. 2, pp. 303-313, Feb. 2013.

[4] W. P. Osborne and M. B. Luntz, "Coherent and noncoherent detection CPFSK," IEEE Trans. Commun., vol. 22, no. 8, pp. 1023-1036, Aug. 1974.

[5] J. B. Anderson, T. Aulin, and C.-E. Sundberg, Digital Phase Modulation. Springer, 1986.

[6] X. Zhang and M. P. Fitz, "Symmetric information rate for continuous phase channel and BLAST architecture with CPM MIMO system," in Proc. IEEE Int. Conf. Commun., May 2003, pp. 3051-3055.

[7] J. Sykora, "Symmetric capacity of nonlinearly modulated finite alphabet signals in MIMO random channel with waveform and memory constraints," in Proc. IEEE Global Telecommun. Conf., Nov. 2004, pp. $425-430$.

[8] A. Lapidoth, A Foundation in Digital Communication. Cambridge University Press, 2009.

[9] B. Hassibi and B. M. Hochwald, "How much training is needed in multiple-antenna wireless links?" IEEE Trans. Inf. Theory, vol. 49, no. 4, pp. 951-963, Apr. 2003.

[10] H. Yang and T. L. Marzetta, "Performance of conjugate and zero-forcing beamforming in large-scale antenna systems," IEEE Select. Areas in Commun., vol. 31, no. 2, pp. 172-179, Feb. 2013. 